\title{
International collaboration generates high quality clusters of pharmaceutical patents
}

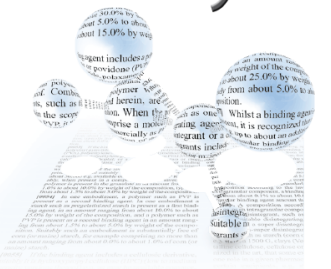

\author{
Christian Moser*,1, Anna M Villa ${ }^{1}$ \& Heinz Mueller ${ }^{1}$ \\ ${ }^{1}$ Swiss Federal Institute of Intellectual Property, Stauffacherstrasse 65, CH-3003 Bern, Switzerland \\ *Author for correspondence: christian.moser@ipi.ch
}

The worldwide active patent portfolio has nearly doubled in numbers and strength since 2000 . The number of active pharmaceutical patent families has tripled in the same time period. The quantitative growth results mostly from a surge of patents from China, half of them classified in A61K36 ('medicinal preparations of undetermined constitution containing material from algae, lichens, fungi or plants'). High-quality patents exhibit a slower growth curve, and cluster within the three areas biologicals; heterocyclic compounds, and cancer drugs. However, the highest concentration of high-quality patents was found when selecting patents listing inventors from at least two out of the five most important countries of origin for pharmaceutical patents: China, EP countries, Japan, South Korea and the USA.

\section{Graphical abstract:}

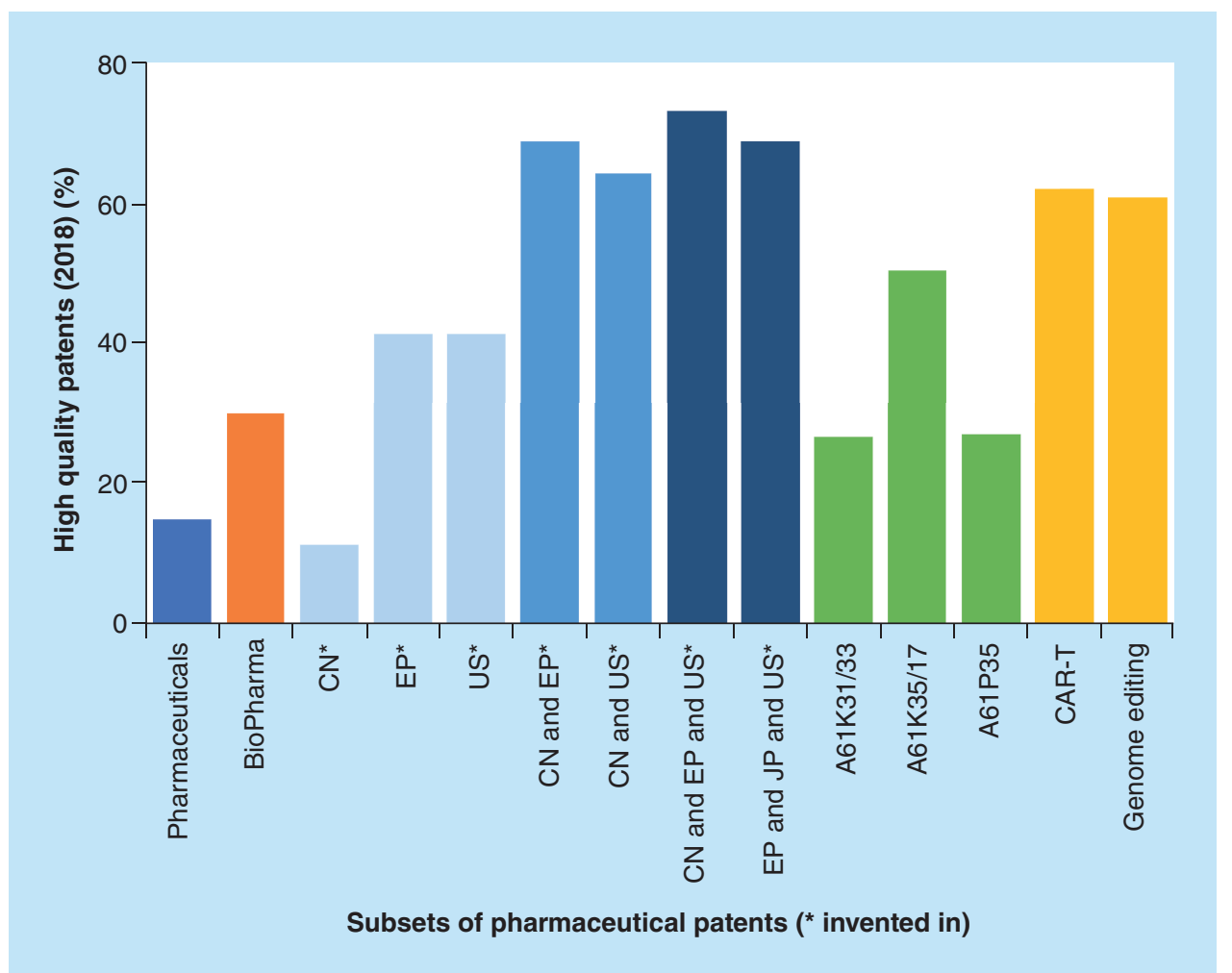

Proportion of high-quality patents in various subsets of pharmaceutical patents. Patents with inventors from more than one country yield higher quality than selected technology fields.

First draft submitted: 4 July 2019; Accepted for publication: 14 August 2019; Published online: 15 October 2019

Keywords: biotechnology $\bullet$ countries $\bullet$ inventors $\bullet$ patent classifications $\bullet$ patent landscape $\bullet$ patent quality parameters • pharmaceuticals 


\section{Methods \& definitions}

\section{Definition of patent sets for analysis}

Pharmaceuticals and biotechnology are two out of 35 technology fields defined by the World Intellectual Property Organization (WIPO) on the basis of their International Patent Classification (IPC) [1]. By expanding these definitions to include also the appropriate Cooperative Patent Classification, a comprehensive set of patents relating to pharmaceuticals (Pharmaceuticals) was generated, and a corresponding set representing Biotechnology. Subsequently, the set BioPharma was created, corresponding to the intersection of Pharmaceuticals and Biotechnology. The fourth set, NonBioPharma, was defined as the patents within Pharmaceuticals, which are not included in BioPharma, thereby ensuring that the sets BioPharma and NonBioPharma are nonoverlapping.

The sets CAR-T and Genome editing resulted from keyword searches using the EPOQUENet software transferred to PatentSight for consolidation to simple patent families.

\section{Statistical analysis}

The patent sets were compiled and analyzed using the patent analysis software PatentSight, using by default the reporting date 31 December 2018. The reporting date refers to the data for determination the count of active patent families. Accordingly, the data for the time courses ranging from 2000 to 2018 are based on the reporting dates at the end of the respective year. Selected datasets were transferred to Microsoft Excel for further analysis and for the creation of the figures.

The Patent Asset Index ${ }^{\top M}$ (PAI) for patent portfolios and Competitive Impact ${ }^{\top M}$ (CI) for individual patent families are qualitative parameters available in the patent analysis software PatentSight [2-4] and further explained in the section definitions. They are an expression of the strength of patent portfolios and further explained in the definitions.

The complete worldwide collection of patents can be divided in value classes according to CI scores. If ten classes are generated, each comprising $10 \%$ of the global set, we speak of CI Deciles (CID). Patent subsets can be analyzed by assessing how many belong into said value classes, thereby providing a concise overview of the quality distribution in the respective set. Patents belonging to the highest decile, for example, CID10, represent the strongest patents, also referrred to as worldclass patents [5-7]. The CID distributions for the sets nonbiopharma and biopharma in Figure 2, and their CID10 shares over time in Figure 3D and E show examples of use.

\section{Definitions}

The patent families represented in all analyses by PatentSight are based on the DOCDB simple patent family definition, which comprises all patents relating to the same common technological contents, as well as the priority documents [8]. Active patent families are defined as patent families granted and in force, or pending at a given time point [2-4].

The origin of inventors and of applicants refers to the addresses of residence provided in the patent applications. Therefore, this information does not necessarily reflect the nationality of the inventors or the geographic R\&D location where the invention actually originated. Patent applications can include multiple inventors or applicants from different countries, therefore a patent family can include more than one country of origin, thereby causing substantial overlaps exceeding 200\% for certain patent sets.

PatentSight operates with the main indicators PAI, CI, Technology Relevance ${ }^{T M}$ (TR), and Market Coverage ${ }^{T M}$ (MC) [2-4]. Briefly explained, for each patent family, a CI value is calculated on the basis of its citations as prior art (TR) and of the juridictions where the patent family is protected (MC). The PAI represents the sum of the CI values of all patents included in a given set of patents, often referred to as portfolio.

The Portfolio size (PS) represents the number of active patent families, in other words, the number of patent families with at least one family member being pending or in force anywhere in the world, at the given timepoint and within the selected set of patents.

The TR is a relative value based on the number of forward citations of the respective patent family, in other words, citations as prior art by patent offices, corrected by a factor which takes into account the age, the technology field and the practices of the citing Patent Office.

The MC is a relative value based on the number of jurisdictions where the patent family is protected, weighted by a factor taking into account the relative strength of the respective economies. 
The $\mathrm{CI}$ is the product of the TR and the MC values for an individual patent family. The CI value calculated for a given patent family is the basis for allocation to the CIDs.

The PAI represents a measure of the overall patent portfolio strength. The PAI is calculated as the sum of the Competitive Impact of all patent families contained in the portfolio.

\section{Introduction}

From 2000 to 2018, the number of active (granted and in force or pending) patent families worldwide has increased from 7.2 to 12.3 million. During the same period, the number of pharmaceutical patents has nearly tripled from 151,000 to 447,000 , accounting for $3.6 \%$ of the global patent portfolio in 2018 (Figure 1A).
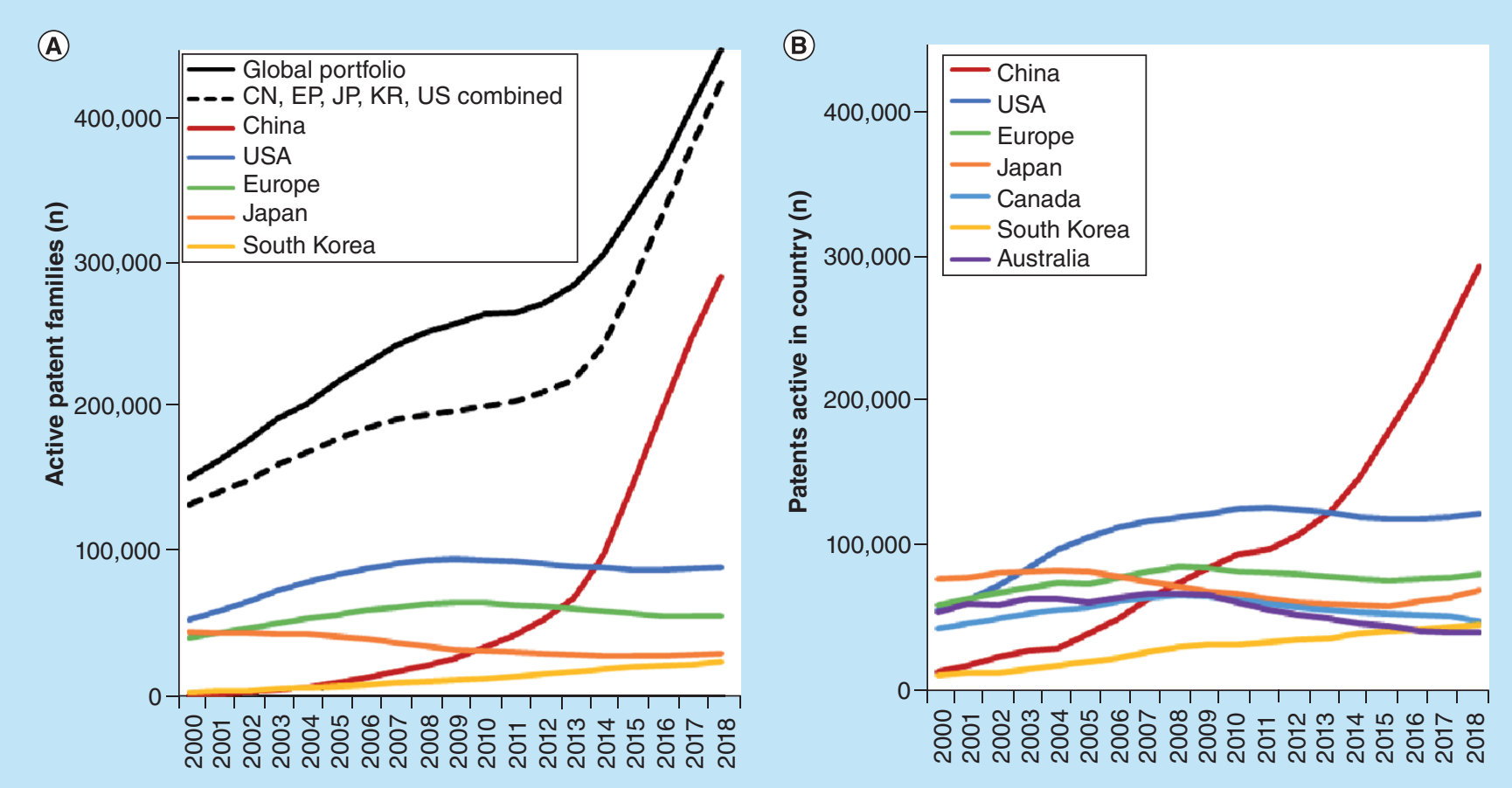

Figure 1. Quantitative dynamics of pharmaceutical patents from 2000 to 2018. (A) Shows the number of active patent families worldwide split by origin of inventors. (B) Shows the patent densities of selected countries, based on the number of active patents per country, irrespective of their origin.

China, the USA, Europe as defined by the European Patent Convention countries [5], Japan, and South Korea are clearly the dominating countries of origin, generating together over $90 \%$ of all pharmaceutical patents (Figure 1A). Since 2000, the number of patents from China has been increasing exponentially. At the same time, the numbers of active patents from the USA, Europe and Japan have remained essentially constant over the past decade, with the result that their share in the global portfolio is shrinking continuously, at least from a quantitative point of view.

The same observations apply with regard to active patents per country (Figure 1B). The inventor countries are also those with highest numbers of active patents, except for Australia and Canada, which have been included due to their high ranking in 2000. Together with the USA, Japan and Europe, Australia and Canada represent countries, where the number of inventions protected is exceeding the number of inventions generated.

An evaluation of the pharmaceutical patents according to the origin of applicants produced a graph nearly identical to Figure $1 \mathrm{~A}$, which relates to the origin of the inventors.

\section{BioPharma versus NonBioPharma}

The PAI for patent portfolios and CI for individual patent families are qualitative parameters available in the patent analysis software PatentSight $[2,3]$ and further explained in the method section. They indicate the strength and quality of a patent portfolio, as shown in Figure 2 for the sets BioPharma and NonBioPharma. While the NonBioPharma patents spread across the CIDs, BioPharma patents cluster more in the higher CIDs. Despite their highly dissimilar CID profiles, the PAI for both sets is concentrated in the high CIDs, in particular CID10. 


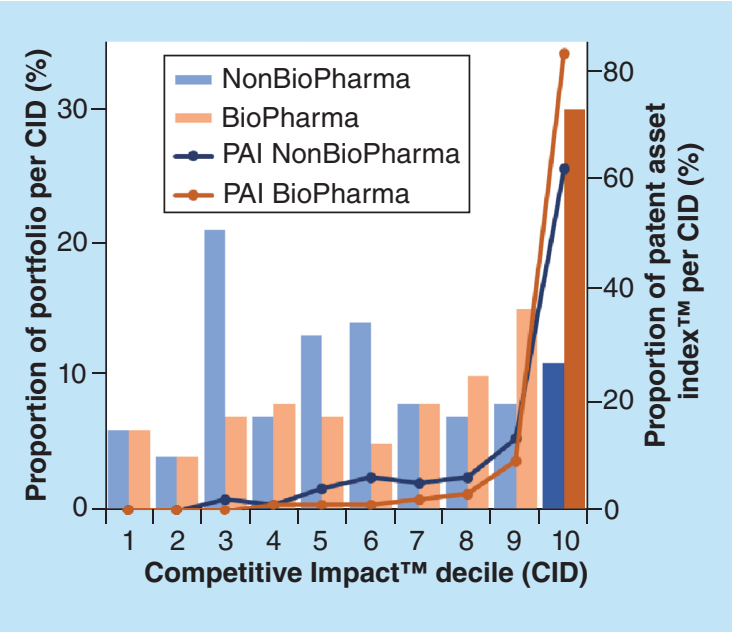

Figure 2. Competitive Impact ${ }^{\top M}$ and Patent Asset Index ${ }^{T M}$ profiles of the sets NonBioPharma and BioPharma for the year 2018. The bars represent the relative number of patents and the lines the PAI per $\mathrm{Cl}$ decile for each of the sets. The darkened bars highlight the patents assigned to CID10 of each set, representing their respective CID10 shares. The data shown refers to the reporting date 31 December 2018. CID: $\mathrm{Cl}$ decile.

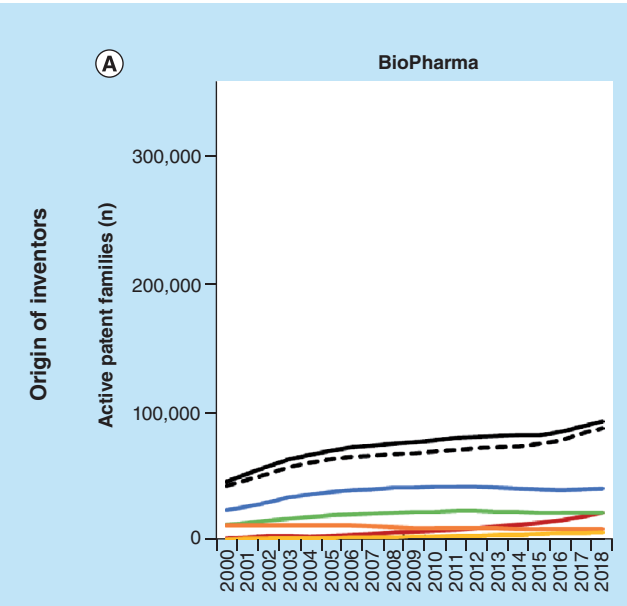

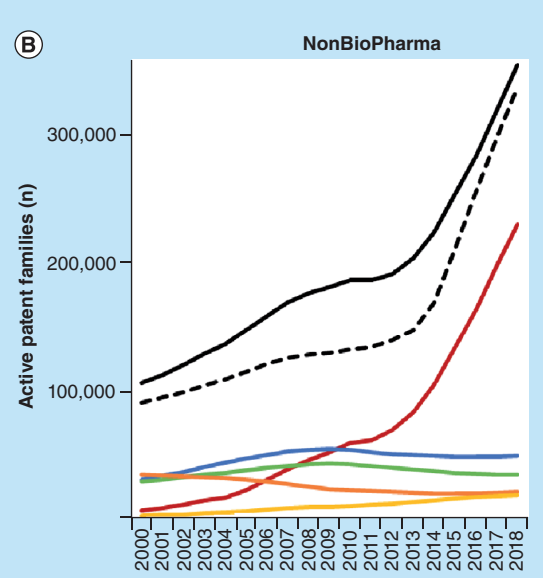

(E)

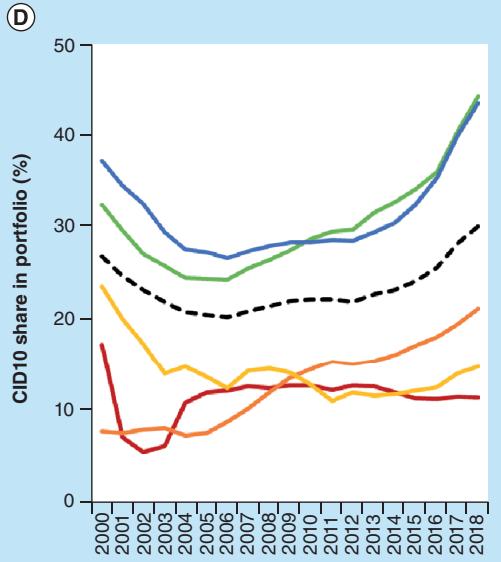

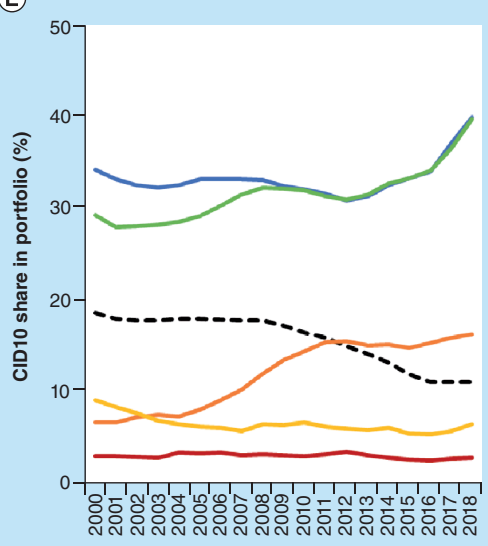

(C)

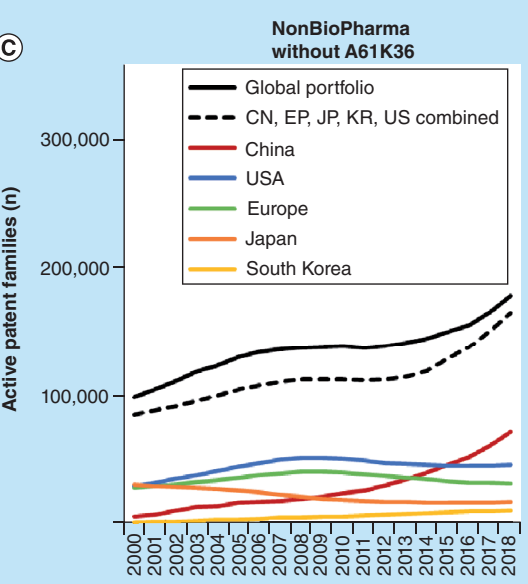

(F)

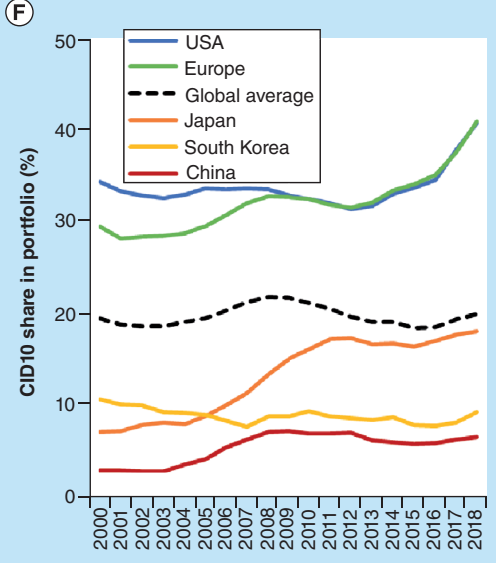

Figure 3. Timelines 2000 to 2018 for the sets BioPharma, NonBioPharma and NonBioPharma without A31K36. The graphs (A), (B) and (C) show the global portfolio as well as the five main countries of origin individually and cumulated. The graphs (D), (E) and (F) depict the CID10 shares of the same countries and the global average.

Therefore, focusing on patent families assigned to CID10 represents a powerful filter for quality. The CID10 filter reduced the number of patent families for the set Pharmaceuticals from 447,000 to 67,000, translating into a CID10 share of $15 \%$ for this set. The CID10 share, in other words, the percentage of CID10 patents within a given subset, facilitates the comparison of multiple subsets of different portfolio sizes and CID distributions with regard 
to their quality. In 2018, the CID10 shares of NonBioPharma and BioPharma were 11 and 30\%, respectively, as depicted by darkened bars in Figure 2.

The timelines for the total subsets BioPharma and NonBioPharma revealed distinct patterns, as shown in Figure 3. For the inventors, the 'country of origin' is determined via the inventor addresses provided in the patent applications. Therefore, the inventor country reflects the country of residence rather than the nationality of the inventors.

The size of BioPharma has doubled since 2000, mostly due to an increase of patents originating from China or Korea (Figure 3A). As of 2018, the BioPharma set was a quarter the size of NonBioPharma. Yet, BioPharma accounted for $40 \%$ of the PAI strength of the entire set Pharmaceuticals (Figure 2). This discrepancy between quantity and quality results from the higher CID10 share of BioPharma for all inventor countries and in global average (Figure 3D vs E), and it is consistent with the higher concentration of BioPharma in the CIDs eight to ten in the CID profile (Figure 2).

The set NonBioPharma has more than tripled in size, with patents originating from China as the obvious main quantitative growth driver of (Figure 3B). The steep increase of Chinese NonBioPharma patents is associated with a low CD10 share, with visible negative impact on the global average (Figure 3E). The quantitative growth resulted largely from a surge of patents classified in A61K36 ('medicinal preparations of undetermined constitution containing material from algae, lichens, fungi or plants'), $98 \%$ of which belong to the NonBioPharma set. As of 2018, the A61K36 set alone comprised 180,000 patent families, representing 40\% of all pharmaceutical patents. However, only $2 \%$ of them are assigned to CID10. Thus, A61K36 patents are clearly a driver for quantitative growth, but not for quality.

In order to eliminate the effects of the dominating A61K36 patents, a refined NonBioPharma set was generated by excluding all A61K36 patents. This subtraction affected the data for China and the global portfolio, but hardly the other countries (Figure $3 \mathrm{C} \& \mathrm{~F}$ ). The resulting global portfolio curve shows a considerably reduced growth rate, more similar to BioPharma.

The patents from the USA and Europe display similar CID10 levels and dynamics in both sectors, BioPharma and NonBioPharma, even though more patents originate from the USA than from EP countries. The distinct increase of the CID10 share in recent years (Figure 3D-F) indicates qualitative growth, a trend which is hardly detectable in the quantitative view of the full set (Figure 3A-C).

\section{Drilling into CID10}

The CID10 subset represents a self-evident starting point for the identification of clusters of high value patents within the set of Pharmaceuticals. Of all patents included in the set pharmaceuticals, more than $95 \%$ feature a A61K classification (medicinal preparations by active principle) and $80 \%$ a A61P classification (medical indications). For the heatmaps shown in Figure 4, the percentage values of the specific subsets relate to the number of patents classified in the respective subclasses, A61K or A61P.

The objective was to find sets of patents, which either are represented in CID10 at higher proportions than in the overall portfolio, or show a significant increase in the number of CID10 patents in recent years.

To this end, the CID10 subsets for the reporting year 2018 were screened for patent classifications meeting these criteria. Figure 4 shows heatmaps of the IPC subclasses A61K and A61P, respectively. However, most of the time multiple patent classifications are assigned to a single patent family, especially if different embodiments and applications of the invention are disclosed. This gives rise to overlaps and redundancies in the heatmap.

As expected, within NonBioPharma, the group A61K36 was prominently represented in the complete set, followed by A61K31 ('medicinal preparations containing organic active ingredients') comprising most if not all small molecule drugs and A61K9 (galenics). This ranking was reversed in the CID10 subset, where A61K31, A61K9 and A61K45 (drug combinations) are the most frequently occurring classifications. When expanding the analysis to lower classification levels, the classification A61K31/33 ('heterocyclic compounds') was the most frequently represented subclass within A61K31, comprising 40,000 patents within CID10, and matching both criteria defined above (high CID proportion: 27\%, recent growth: 25\% increase in numbers since 2014).

In contrast to NonBioPharma, BioPharma revealed no significant differences between the entire set and the CID10 subset, with A61K39 (antibodies and antigens) and A61K38 (peptides) as the most represented classifications. A61K31 was also prominently present in BioPharma, presumably due to nucleic acid compounds being classified in A61K31/70 (sugars) and because of combinations of biologicals with small molecules, in particular 


\begin{tabular}{|c|c|c|c|c|c|}
\hline & & \multicolumn{2}{|c|}{ NonBioPharma } & \multicolumn{2}{|c|}{ Biopharma } \\
\hline & & $\begin{array}{l}\text { All patents in } \\
\text { set }(\%)\end{array}$ & $\begin{array}{c}\text { CID10 patents } \\
(\%)\end{array}$ & $\begin{array}{l}\text { All patents in } \\
\text { set (\%) }\end{array}$ & \begin{tabular}{|c} 
CID10 patents \\
$(\%)$
\end{tabular} \\
\hline \multirow{13}{*}{ 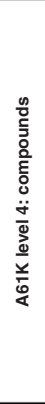 } & A61K 9: Medicinal preparations characterised by special physical form & 29 & 34 & 12 & 17 \\
\hline & A61K 31: Medicinal preparations containing organic active ingredients & 45 & 78 & 30 & 37 \\
\hline & \begin{tabular}{|l} 
A61K 33: Medicinal preparations containing inorganic active ingredients \\
\end{tabular} & 8 & 6 & 2 & 2 \\
\hline & A61K 35: Medicinal preparations containing materials or reaction products thereof with undetermined constitution & 18 & 6 & 20 & 23 \\
\hline & A61K 36: Medicinal preparations of undetermined constitution containing material from algae, lichens, fungi or plants, or derivatives thereof, & 52 & 13 & 4 & 2 \\
\hline & A61K 38: Medicinal preparations containing peptides & 3 & 7 & 41 & 42 \\
\hline & A61K 39: Medicinal preparations containing antigens or antibodies (materials for immunoassay) & 1 & 3 & 37 & 46 \\
\hline & \begin{tabular}{|l} 
A61K 41: Medicinal preparations obtained by treating materials with wave energy or particle radiation (takes precedence) \\
\end{tabular} & 0 & 0 & 1 & 1 \\
\hline & A61K 45: Medicinal preparations containing active ingredients not provided for in groups & 17 & 36 & 16 & 23 \\
\hline & A61K 47: Medicinal preparations characterised by the non-active ingredients used, e.g. carriers or inert additives; & 5 & 8 & 16 & 25 \\
\hline & A61K 48: Medicinal preparations containing genetic material which is inserted into cells of the living body to treat genetic diseases; gene therapy & 2 & 3 & 17 & 19 \\
\hline & A61K 49: Preparations for testing in vivo & 0 & 1 & 5 & 6 \\
\hline & A61K 51: Preparations containing radioactive substances for use in therapy or testing in vivo & 1 & 2 & 3 & 4 \\
\hline \multirow{23}{*}{ 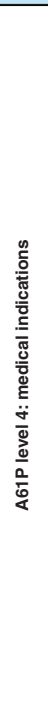 } & A61P 1: Drugs for disorders of the alimentary tract or the digestive system & 19 & 21 & 14 & 19 \\
\hline & A61P 3: Drugs for disorders of the metabolism (of the blood or the extracellular fluid) & 12 & 23 & 13 & 18 \\
\hline & A61P 5: Drugs for disorders of the endocrine system & 2 & 7 & 4 & 6 \\
\hline & A61P 7: Drugs for disorders of the blood or the extracellular fluid & 6 & 11 & 9 & 12 \\
\hline & A61P 9: Drugs for disorders of the cardiovascular system & 13 & 23 & 15 & 18 \\
\hline & A61P 11: Drugs for disorders of the respiratory system & 10 & 16 & 8 & 13 \\
\hline & A61P 13: Drugs for disorders of the urinary system (diuretics) & 6 & 13 & 6 & 10 \\
\hline & A61P 15: Drugs for genital or sexual disorders (for disorders of sex hormones); contraceptives & 8 & 8 & 4 & 6 \\
\hline & A61P 17: Drugs for dermatological disorders & 12 & 16 & 10 & 15 \\
\hline & A61P 19: Drugs for skeletal disorders & 9 & 16 & 11 & 15 \\
\hline & A61P 21: Drugs for disorders of the muscular or neuromuscular system & 2 & 7 & 5 & 8 \\
\hline & A61P 23: Anaesthetics & 0 & 1 & 0 & 0 \\
\hline & A61P 25: Drugs for disorders of the nervous system & 15 & 31 & 16 & 21 \\
\hline & \begin{tabular}{|l} 
A61P 27: Drugs for disorders of the senses \\
\end{tabular} & 5 & 12 & 6 & 10 \\
\hline & A61P 29: Non-central analgesic antipyretic or antiinflammatory agents, e.g antirheumatic agents; non-steroidal antinflammatory drugs (NSAIDs) & 14 & 24 & 13 & 18 \\
\hline & A61P 31: Antiinfectives, i.e. antibiotics, antiseptics, chemotherapeutics & 16 & 22 & 28 & 28 \\
\hline & A61P 33: Antiparasitic agents & 2 & 4 & 3 & 4 \\
\hline & A61P 35: Antineoplastic agents & 17 & 35 & 40 & 49 \\
\hline & A61P 37: Drugs for immunological or allergic disorders & 8 & 18 & 21 & 30 \\
\hline & A61P 39: General protective or antinoxious agents & 5 & 3 & 2 & 2 \\
\hline & \begin{tabular}{|l|l|} 
A61P 41: Drugs used in surgical methods, e.g. surgery adjuvants for preventing adhesion or for vitreum substitution \\
\end{tabular} & 0 & 1 & 0 & 1 \\
\hline & A61P 43: Drugs for specific purposes, not provided for in groups & 9 & 36 & 23 & 35 \\
\hline & A61Pmiss: Specific therapeutic activity of chemical compounds or medicinal preparations & 3 & 17 & 6 & 13 \\
\hline
\end{tabular}

Figure 4. Heatmaps of $A 61 \mathrm{~K}$ and A61P International Patent Classification level 4 classifications. The heatmap in green refers to the entire sets whiles the heatmap in red represents the CID10 subsets of NonBioPharma and BioPharma. The data shown refers to the reporting date 31 December 2018.

CID: $\mathrm{Cl}$ decile.

with molecules classified in A61K31/33 (heterocyclic active principles). Finally, A61K35 including cell therapies and probiotics are highest in CID10, showing an overall high quality of these technologies.

The aspect of medical indication showed a broad distribution among the subclasses and less discrepancies between NonBioPharma and BioPharma: The group A61P35 ('antineoplastic agents') seems well represented in both sets, followed by A61P43 ('drugs for specific purposes'). The A61P35 patents feature 27\% CID10 share, and a 55\% increase in numbers since 2014. The medical indication heatmap identified additional classifications with increased shares in CID10 compared with the entire set, such as A61P37 ('drugs for immunological disorders'), A61P27 ('drugs for disorders of the nervous system') or A61P9 ('drugs for disorders of the cardiovascular system').

\section{International co-inventions}

Interestingly, the highest CID10 shares were found in subsets generated by combining origins of inventors. When filtering for pharmaceutical patents, which list inventors from China, the USA and Europe, a set of 1600 patent families resulted, featuring a CID10 share of 73\%. Notably, the average market coverage and the technology relevance values for this set were both slightly higher than the corresponding average values of all 67,000 CID10 patents within Pharmaceuticals. Therefore, the strength of the international co-inventions does not result from broader patent filing only, but equally from technological excellence. 


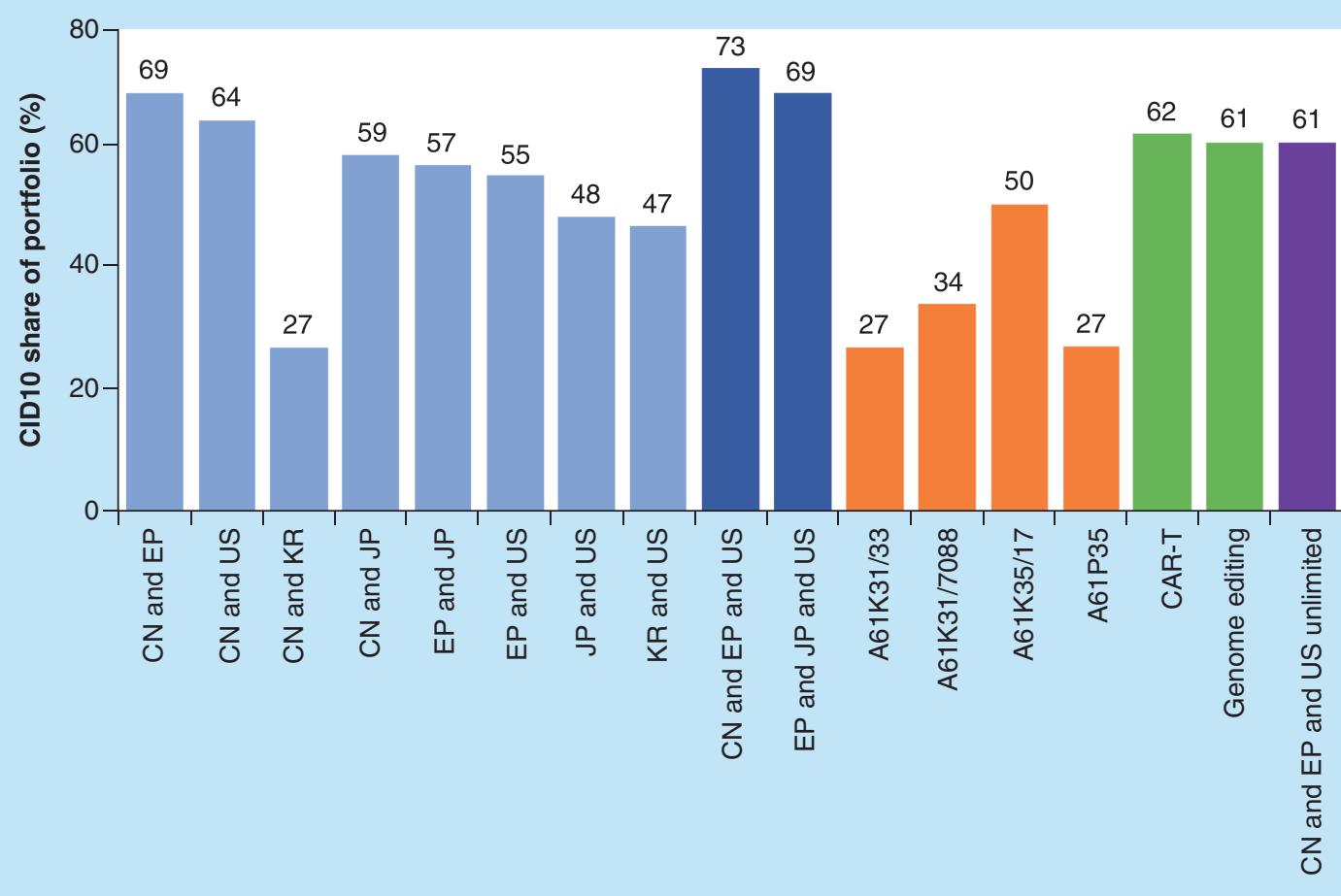

Figure 5. CID10 shares of selected patent subsets within the set pharmaceuticals. The sets were compiled by selection for individual or multiple origins of inventors, by IPC classifications, or by keyword search concepts (CAR-T, Genome editing). The rightmost bar (CN and EP and US unlimited) represents 6000 patent families without restriction to Pharmaceuticals. The data shown refers to the reporting date 31 December 2018.

IPC: International Patent Classification.

The combination of China and Europe comprising 2000 patent families resulted in a CID10 share of 69\%, which is higher than the CID10 share of selected benchmark technology fields shown in Figure 5. The patents relating to the biotechnology fields CAR-T (cell therapies based on chimeric antigen receptors) and Genome editing (including CRISPR/Cas, TALENs and $\mathrm{ZnF}$ technologies) were used to establish benchmark values for the CID10 share, yielding values of 62 and $61 \%$, respectively (Figure 5, orange bars). These sets have been recently compiled based on keyword searches [6], each comprising less than 3000 patent families, a portfolio size comparable to the co-inventor sets.

The permutations with two or three inventor country combinations for the five countries (China, Europe, Japan, South Korea and USA) revealed in all cases higher CID10 shares for the combinations than for either of the countries alone. However, the set sizes of the combinations varied between less than 30 and over 13,000 patents.

A search without any technological limitation for co-inventions from China, Europe and the USA retrieved a set of over 7000 patent families with a CID10 share of 61\% (Figure 5, purple bar), suggesting that high percentages in CID10 for co-inventions are not restricted to pharmaceuticals.

\section{Summary \& conclusion}

Beyond purely quantitative patent statistics, qualitative parameters are increasingly used to generate meaningful patent landscapes [9-12]. The software PatentSight used in the present study [2-4] is one of several tools on the market, which combine enhanced patent databases with an advanced quality analysis software $[9-11,13]$. Such tools are useful for investigating large sets of patent documents in order to assess and compare different patent portfolios, or to screen for trends and relationships [5-7,14-20].

The present study has evaluated the large and heterogeneous set of patents in the field of pharmaceuticals with a top-down approach. The objective was to provide an overview of the entire set with regard to quantitative and qualitative aspects, and to split it into meaningful subsets with distinct properties. 
In summary, our observations show that patents from Chinese, and to a lesser extent, from South Korean inventors have been driving the quantitative growth in the past decade. After a consolidation phase, patents from other important countries of origin, in other words, the USA, Europe and Japan, show indications of growth during the past 4 years, but in contrast to China and South Korea, rather in quality than in quantity. Pharmaceutical patents involving biotechnology account for only $20 \%$ of all pharmaceutical patents, but represent $40 \%$ of their total strength measured according to the PatentSight metrics. Notably, this observation applies globally, in other words, all countries examined in this study show the same pattern.

In a second step, clusters of high-value patents were filtered by focusing on the patents assigned to the highest CID10. The resulting subsets - cancer therapies, certain categories of biologicals and heterocyclic compounds do per se not represent unexpected results. However, each of these sets still comprises 10,000 of patent families each. Smaller, more homogeneous clusters of high value patents could be identified within sets, requiring further drilling, by exploring lower classification levels, or by means of keyword filters or artificial intelligence tools, such as semantic analysis or text mining $[11,12]$.

Interestingly, a combination of countries of origin has yielded sets with higher CID10 shares when compared with the explorations of specific technology areas like CAR-T or genome editing. This finding may further support the notion that international collaboration is an important factor in yielding high-value innovation in pharmaceuticals [20]. Remarkably, our data indicate that this observation is not limited to pharmaceuticals but seems to be a general phenomenon. International collaboration is generally considered an important success factor in research [21,22], and scientific publications by international co-authors were shown to yield higher average citation impact than articles by domestic authors [23].

As regards the contributions from individual countries, an earlier study within the biotechnology field [6] reported CID10 shares in the range of $40-50 \%$ for Denmark, Switzerland, the UK and The Netherlands, while the USA reached $35 \%$.

The present study focused on the origins of inventors and technology fields in relation to quantitative and qualitative parameters. Obviously, the patent sets and subsets presented here constitute a well-defined starting point for the investigation of further aspects of interest, for example, the ownership structure and distribution between corporate entities, public research organizations or governments.

\section{Future perspective}

Under the assumption that the current trends will persist for the next decade, patent families from China will increasingly dominate the global patent landscape, initially in terms of numbers, but with some delay also in terms of portfolio strength.

In the sector NonBioPharma, Chinese patent families dominate already, while in BioPharma China might catch up in numbers with the USA within the next 5 years, based on the current growth rate. This prediction is realistic, because the patent portfolios from the USA, the EP countries and Japan show no trend for quantitative growth and are likely to remain in the same range as in the past decade (Figure 3A-C).

The CID10 share of the Chinese portfolio has remained constant, despite of the massive quantitative growth. As a result, the number of CID10 patents from China and the overall Chines portfolio strength is rapidly increasing, with no indications that this trend will change in the near future. In contrast, the CID10 shares but not the size of the portfolios from the USA and the EP countries have increased substantially in the past 5 years, but it is difficult to predict for how long and to which level this trend can persist (Figure 3D-F). Thus, based on the current trends, the potential for further increase of the overall portfolio strength seems substantially higher for China than for the USA, Europe and Japan combined.

Supplementary data

To view the supplementary data that accompany this paper please visit the journal website at: www.futurescience.com/doi/suppl/10.4155/ppa-2019-0017 
Financial \& competing interests disclosure

All three authors are employees of the Swiss Federal Institute of Intellectual Property (IPI) and have written the present manuscript in their functions as employees of the IPI. No financial or material support was received from other entities. The authors have no other relevant affiliations or financial involvement with any organization or entity with a financial interest in or financial conflict with the subject matter or materials discussed in the manuscript apart from those disclosed.

No writing assistance was utilized in the production of this manuscript.

\section{Executive summary}

- The number of active patents relating to pharamceuticals has tripled since 2000

- The bulk of the growth results from a surge of patents from China, half of them classified in IPC A61K36 ('medicinal preparations of undetermined constitution containing material from algae, lichens, fungi or plants') featuring a low quality based on their average Competitive Impact ${ }^{\mathrm{TM}}$.

- Over $90 \%$ of all pharmaceutical patents originate from China, EP countries (i.e., the EPC member states), Japan, South Korea or the USA.

- The number of active patents from the US, EP countries and Japan has remained largely constant for the past decade, but their average quality has increased.

- Biologicals, in other words, patents relating to both pharmaceuticals and biotechnology, account for $20 \%$ of the global pharamceutical patent portfolio, but represent $40 \%$ of the portfolio strength measured by Patent Asset Index ${ }^{\top \mathrm{M}}$.

- High-quality patents cluster within the sectors biologicals (IPC classifications A61K38 and A61K39), cancer drugs (IPC classification A61P35) and heterocyclic compounds (IPC classification A61K31/33).

- The highest proportion of high-quality patents is found in subsets of patents listing at least two out of the five major countries of origin for Pharmaceuticals.

\section{References}

Papers of special note have been highlighted as: • of interest; $\bullet \bullet$ of considerable interest

1. Anonymous WIPO. World Intellectual Property Indicators 2017. Geneva: World Intellectual Property Organization (2017). https://www.wipo.int/edocs/pubdocs/en/wipo_pub_941_2017.pdf pdf

- Definition of 35 technology fields based International Patent Classifications.

2. Omland N. Valuing patents through indicators. In: The Economic Valuation of Patents: Methods and applications. Munari F, Oriani R (Eds). Edward Elgar Publishing, Cheltenham, UK, 169-204 (2011).

-• Theoretical basis of the PatentSight metrics.

3. Ernst H, Omland N. The patent asset index-A new approach to benchmark patent portfolios. World Patent Information 33(1), 34-41 (2011).

-. Empirical validation of the PatentSight metrics for chemistry patents.

4. The Patent Asset Index ${ }^{\top M}$. An objective measure of global technological strength and influence. www.patentsight.com/en-us/patent-asset-index

5. Gramke K, Glauser C. Patent Activities Relating to Digitalisation Technologies (in German), short report mandated by the Swiss Government (2017). www.sbfi.admin.ch/sbfi/en/home/services/publications/data-base-publications/digitalisation-technologies

- Definition of patents assigned to CID10 as 'world-class patents'.

6. Mueller H, Moser C. Swiss biotech patents: a history of quantitative and qualitative growth. Swiss Biotech Report 2018 12-13 (2018). www.swissbiotech.org/report

7. Moser C. Trends in biotechnology: visualizing Swiss innovation through patent landscape analysis. Swiss Biotech Report 2019 11-13 (2019). www.swissbiotech.org/report

8. Patent families. www.epo.org/searching-for-patents/helpful-resources/first-time-here/patent-families

9. Oldham P, Kitsara I. WIPO manual on open source tools for patent analytics (2016). www.wipo-analytics.github.io

-. Review of noncommercial, open source tools for patent analytics.

10. Aristodemou L, Tietze F. A literature review on the state-of-the-art on intellectual property analytics. Cent. Technol. Manag. Work. Pap. Ser. https://doi.org/10.17863/CAM.13928 (2017).

11. Lupu M. Information retrieval, machine learning, and natural language processing for intellectual property information. World Patent Information 49, A1-A3 (2017).

12. Aristodemou L, Tietze F. The state-of-the-art on Intellectual Property Analytics (IPA): a literature review on artificial intelligence, machine learning and deep learning methods for analysing intellectual property (IP) data. World Patent Information 55, 37-51 (2018). 
-. Review of state-of-the-art IP analytics.

13. The European Patent Convention. www.epo.org/law-practice/legal-texts/epc.html

14. Qu J, Lu J, Hu Y. Research and development of anti-Parkinson's drugs: an analysis from the perspective of technology flows measured by patent citations. Expert Opin. Ther. Pat. 29(2), 127-135 (2019).

15. Lahoti G, Porter AL, Zhang C, Youtie J, \& Wang B. Tech mining to validate and refine a technology roadmap. World Patent Information 55, 1-18 (2018).

16. Guderian CC. Identifying emerging technologies with smart patent indicators: the example of smart houses. Int. J. Innovation Technol. Manage. 16(2), 1-24 (2019).

17. Jiang Q, Luan C. Diffusion, convergence and influence of pharmaceutical innovations: a comparative study of Chinese and U.S. patents. Globalization Health 14(1), 92 (2018).

18. Dierks RML, Bruyère $\mathrm{O}$, Reginster JY. Critical analysis of valuation and strategical orientation of merger and acquisition deals in the pharmaceutical industry. Expert Rev. Pharmacoecon. Outcomes Res. 18(2), 147-160 (2018).

19. Fankhauser M, Moser C, Nyfeler T. Patents as early indicators of technology and investment trends: analyzing the microbiome space as a case study. Front. Bioeng. Biotechnol. 6, 84 (2018).

20. Tufféry P. Accessing external innovation in drug discovery and development. Expert Opin. Drug Discov. 10(6), 579-589 (2015).

21. Tijssen RJ, Winnink JJ. Capturing 'R\&D excellence': indicators, international statistics, and innovative universities. Scientometrics 114(2), 687-699 (2018).

- Assessing R\&D excellence of institutions and countries via scientific publications and frequently cited patents.

22. Schmidt EK, Graversen EK. Persistent factors facilitating excellence in research environments. Higher Education 75(2), 341-363 (2018).

- Scientific publicationsby international co-authors generate a higher average citation impact than domestic authors only.

23. Adams J, Gurney KA. The implications of international research collaboration for UK universities. Digital Research Report (2016). www.digital-science.com/digital-research-reports/digital-research-report-the-implications-of-international-researchcollaborations-for-uk-universities/ 